\title{
Proposal of a Japanese-speech-synthesis Method with Dimensional Representation of Emotions based on Prosody as well as Voice-quality Conversion
}

\author{
Shoichi TAKEDA*, Yoshiki KABUTA**, Tomohiro INOUE* and Masashi HATOKO** \\ * Faculty of Biology-Oriented Science and Technology, Kinki University, \\ 930 Nishi-Mitani, Kinokawa-shi, Wakayama 649-6493, Japan \\ ** Graduate School of Biology-Oriented Science and Technology, Kinki University, \\ 930 Nishi-Mitani, Kinokawa-shi, Wakayam, 649-6493, Japan
}

\begin{abstract}
This paper proposes a Japanese speech synthesis system that is capable of expressing variable degrees of emotions based on prosody as well as voice-quality conversion. Among voice-quality features, we find that the spectral tilts depend on the type and degree of emotion. Up to date, we have introduced a spectral-tilt conversion rule into our speech-synthesis system. From our previous analyses, we found that the spectral-tilt quantities increased as the degrees of "anger", "joy", and "crying-type (hot) sadness" increased. On the other hand, the spectral-tilt quantities were found to decrease as the degree of "dispirited-and-whispering-type (cold) sadness" increased. We formulate a transfer function that converts spectral-tilt quantities of "neutral" speech to those of emotional speech in various degrees. The prosody-conversion rules are also determined based on our previous findings. Informal listening to syntheticspeech samples converted by the proposed method gives us impressions of those similar to natural emotional speech and the differences depending on the degrees of emotions are recognizable.
\end{abstract}

Keywords: Speech Synthesis, Emotional Expression, Degree of Emotion, Voice Quality, Spectral Tilt

\section{INTRODUCTION}

Owing to recent advancement of speech technology, synthetic speech has remarkably improved its quality and is being used in various fields.

The current synthetic speech applied in practical fields, such as electronic dictionaries, e-mail reading, etc., is, however, mostly non-expressive. It is therefore necessary to develop a technique to synthesize expressive speech if we want to extend its application more widely.

Among expressive speech, we have placed a focus on emotional speech such as "anger", "joy", "sadness", "gratitude", etc. As the first step, we analyzed the prosodic features of various emotional expressions to achieve more natural-sounding rule-based synthetic speech $[5,6]$.

The importance of research on emotional expressions has been widely recognized. Such relevant works include Scherer [14], Banse and Scherer [1], Schröder and Grice [15], Juslin and Laukka [8]. Among them, however, reports on Japanese speech synthesis were few [3].

In early studies, researchers analyzed rough features of typical emotional expressions such as "joy," "anger," etc. and/or synthesized emotional speech based on these features $[10,11]$. These studies, however, gave a mere rough paradigm of emotional expressions such as "joy", "sadness", "anger", etc. They therefore left further studies to give rules to express detailed emotional nuances.

We have been taking more detailed approach instead of investigating various types of typical emotional expressions roughly. We have divided such emotions as "anger", "joy", and "sadness" into four degrees: "neutral", "light", "medium", and "strong" and features of each degree have been analyzed [5,6]. Here, "a degree of emotion" refers to the intensity factor of an emotion and it has so far been determined subjectively by the speaker.

There are still few reports on such research, in which each emotional expression was divided into several degrees and it was pursued how the features differed depending on the degree of emotion [7]. Recently, Bänziger and Scherer investigated the features of emotional expressions in languages other than Japanese in terms of the degree of emotion [2].

The quality of speech synthesized based on only prosodic features, however, did not sufficiently express emotions. We learned that not only prosodic features but also some other features must be used to express emotions. 
Among such features, voice-quality [22] features were investigated.

Some of the most important factors that affect voice quality of such emotional speech may be noise in speech, specifically in excitation, spectral shapes, i.e., spectral tilt and depth between the peaks and dips of the spectrum, etc.

With regard to noise levels of speech, we measured the noise levels of the predictive residual signal of speech that expressed several degrees of each emotion and clarified quantitatively that the noise levels differed depending on the type and degree of emotion [19].

We have also done research on the features of the spectral-tilt quantities of speech. So far, many researchers have done work on spectral-tilt features $[9,12]$. What is novel in our research is that we have clarified the spectraltilt features of multiple degrees of emotions in Japanese speech. Our previous paper reported the spectral-tilt features of "anger", "joy" [20], and "sadness" [16] groups.

Based on these findings, we have further proposed a method for converting a spectral-tilt quantity for "neutral" speech to that for several types and degrees of emotional speech to achieve speech synthesis with dimensional representation of emotions [21].

This paper proposes such a Japanese speech synthesis system based on prosody as well as voice-quality conversion and demonstrates several examples of implementation results.

\section{OVERVIEW OF EMOTIONAL SPEECH SYNTHESIS SYSTEM DESIGN}

Based on our findings clarified so far, we propose an emotional speech synthesis system as shown in Figure 1,

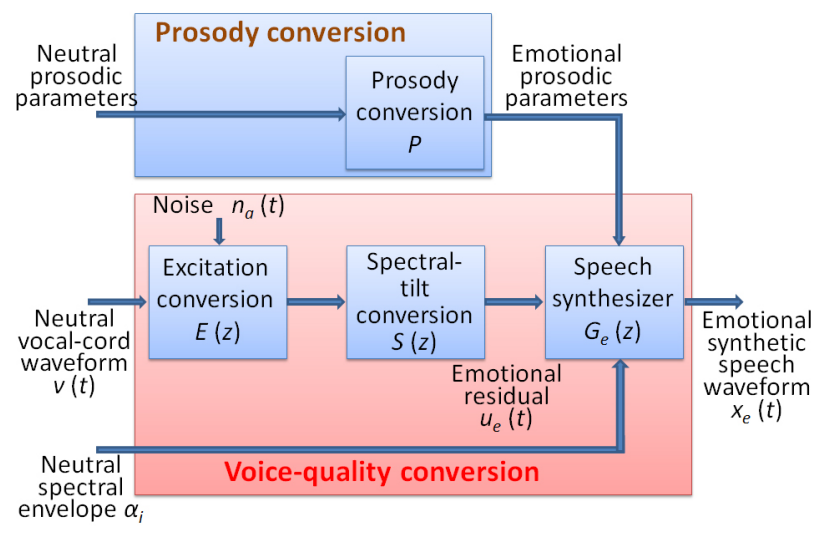

Figure 1: Overview of speech synthesis system capable of expressing variable degrees of emotions. consisting of prosody conversion as well as voice-quality conversion functions. This system can produce multiple degrees of emotional speech from "neutral" prosodic as well as voice-quality parameters. This system will be applicable to corpus-based speech synthesis including statistical parametric speech synthesis that is being widely used [13]. This time, however, we try to apply our proposed method to our available system, a conventional residual-excited PARCOR synthesis system [18].

Prosody conversion can be achieved by using the knowledge of prosodic features of emotional speech $[5,6]$.

In voice-quality conversion, an excitation conversion method is being developed. Spectral-tilt conversion can be achieved if we apply knowledge of the spectral-tilt features that have been accumulated so far $[16,20]$. Among the voice-quality conversion depicted in Figure 1, this paper focuses on spectral-tilt conversion.

Several examples of rules for emotional-speech synthesis using the prosody as well as voice-quality conversions, specifically spectral-tilt conversion, will be described in the latter section.

\section{PROSODIC FEATURES $[5,6]$}

As prosodic-feature parameters, speech rate, fundamental frequency, and speech power were analyzed for speech samples uttered by four announcers as well as four radio actors/actresses. The results showed that the speaker-independent properties of "anger" and "joy" were that the speakers raised both fundamental frequency and speech power and those of "sadness" were that they reduced speech rate, with the increase of the degrees of the emotions. Furthermore, the properties of "sadness" were found to be divided into two types: one called "hot sadness" was that the speakers raised speech power, often also raising fundamental frequency and the magnitude of accent command [4], and the other called "cold sadness" was that they reduced speech power, often also reducing fundamental frequency and the magnitude of accent command, with the increase of the degrees of the emotions.

Table 1 summarizes the prosodic features of various emotions in speech uttered by announcers compared to those of neutral speech. In the same way, Table 2 summarizes those uttered by radio actors/actresses. It should be noted that the tendencies of maximum speech powers for radio actors/actresses are less reliable due to their large body movements during utterances for expressing emotions (denoted by parentheses). 
Table 1: Summary of the prosodic features of various emotions (speakers: announcers).

\begin{tabular}{|c|c|c|c|c|c|c|}
\hline \multirow[b]{2}{*}{ Emotion } & \multirow[b]{2}{*}{ Gender } & Temporal & \multicolumn{3}{|c|}{ Fundamental frequency } & \multirow{2}{*}{\begin{tabular}{|c} 
Intensity \\
$\begin{array}{c}\text { Maximum speech } \\
\text { power }\end{array}$
\end{tabular}} \\
\hline & & $\begin{array}{l}\text { Mean speech } \\
\text { rate }\end{array}$ & $\begin{array}{c}\text { Magnitude of } \\
\text { phrase command } \\
A_{\mathrm{p}}\end{array}$ & $\begin{array}{c}\text { Magnitude of } \\
\text { accent command } \\
A_{\mathrm{a}} \\
\end{array}$ & $\begin{array}{c}\text { Maximum } \\
\text { fundamental } \\
\text { frequency } F_{0 \max }\end{array}$ & \\
\hline \multirow[t]{2}{*}{ Anger } & Male & $\begin{array}{l}\text { Increase (anger) } \\
\text { Reduction (fury) }\end{array}$ & Reduction & \multirow[t]{2}{*}{ Increase } & \multirow[t]{2}{*}{ Increase } & \multirow[t]{2}{*}{ Increase } \\
\hline & Female & Increase & Increase & & & \\
\hline \multirow{2}{*}{ Joy } & Male & Reduction & \multirow{2}{*}{ No tendency } & \multirow{2}{*}{ Increase } & \multirow{2}{*}{ Increase } & \multirow{2}{*}{ Increase } \\
\hline & Female & Increase & & & & \\
\hline \multirow{2}{*}{ Sadness } & Male & \multirow{2}{*}{ Reduction } & \multirow{2}{*}{ No tendency } & \multirow{2}{*}{ No tendency } & \multirow{2}{*}{ No tendency } & Increase \\
\hline & Female & & & & & Reduction \\
\hline
\end{tabular}

Table 2: Summary of the prosodic features of various emotions (speakers: radio actors/actresses).

\begin{tabular}{|c|c|c|c|c|c|c|}
\hline \multirow[b]{2}{*}{ Emotion } & \multirow[b]{2}{*}{ Gender } & $\begin{array}{l}\text { Temporal } \\
\text { structure }\end{array}$ & \multicolumn{3}{|c|}{ Fundamental frequency } & \multirow{2}{*}{\begin{tabular}{|c|} 
Intensity \\
$\begin{array}{c}\text { Maximum speech } \\
\text { power }\end{array}$
\end{tabular}} \\
\hline & & $\begin{array}{l}\text { Mean speech } \\
\text { rate }\end{array}$ & $\begin{array}{c}\text { Magnitude of } \\
\text { phrase command } \\
A_{\mathrm{p}}\end{array}$ & $\begin{array}{c}\text { Magnitude of } \\
\text { accent command } \\
A_{\mathrm{a}}\end{array}$ & $\begin{array}{l}\text { Maximum } \\
\text { fundamental } \\
\text { frequency } F_{0 \max }\end{array}$ & \\
\hline \multirow{2}{*}{ Anger } & Male & \multirow{2}{*}{ Reduction } & Increase & \multirow{2}{*}{ Increase } & \multirow{2}{*}{ Increase } & \multirow{2}{*}{ (Increase) } \\
\hline & Female & & No tendency & & & \\
\hline \multirow[t]{2}{*}{ Joy } & Male & \multirow{2}{*}{ Reduction } & \multirow{2}{*}{ Reduction } & \multirow{2}{*}{ Increase } & \multirow{2}{*}{ Increase } & \multirow{2}{*}{ (Increase) } \\
\hline & Female & & & & & \\
\hline \multirow{2}{*}{ Sadness } & Male & \multirow{2}{*}{ Reduction } & \multirow{2}{*}{ Reduction } & \multirow{2}{*}{ No tendency } & \multirow{2}{*}{ No tendency } & \multirow{2}{*}{ (Increase) } \\
\hline & Female & & & & & \\
\hline
\end{tabular}

\section{VOICE-QUALITY FEATURES}

\subsection{Emotion-dependent Noise Levels [19]}

We paid attention to the noise level of the glottal-flow waveform. We adopted the AR model and measured the noise levels of the predictive residual signal of speech that expressed each emotion. To measure a relative noise level to the signal level, the "noise-to-signal (N/S) ratio" was introduced.

The analysis results showed that the relative noise levels in the residual-waveform spectra were different, i.e., the N/S ratio of each emotion was larger in the order of "anger" > "sadness" ₹ "neutral" > "joy" by approximately $4[\mathrm{~dB}]$. These results were speaker- and word-independent.

\subsection{Emotion-dependent Spectral-tilt Quantities 4.2.1 Definition of a spectral tilt [20]}

Kasuya $e t$ al. defined a spectral-tilt parameter $T L$ as a difference between the peak dB-power level and the dB-power level at 2-3 [kHz] of the spectrum of the source waveform [9]. In this paper, we defined a spectral-tilt parameter in a different way so that the parameter matched our proposed speech synthesis system described in Figure 1.

Figure 2 shows power spectra and their regression lines obtained by the least squared method. In the figure, the blue curve is the spectrum at the first /a/ in the word "atonomatsuri" with "neutral" emotion and the red one is the spectrum at the same part of the word with "fury"

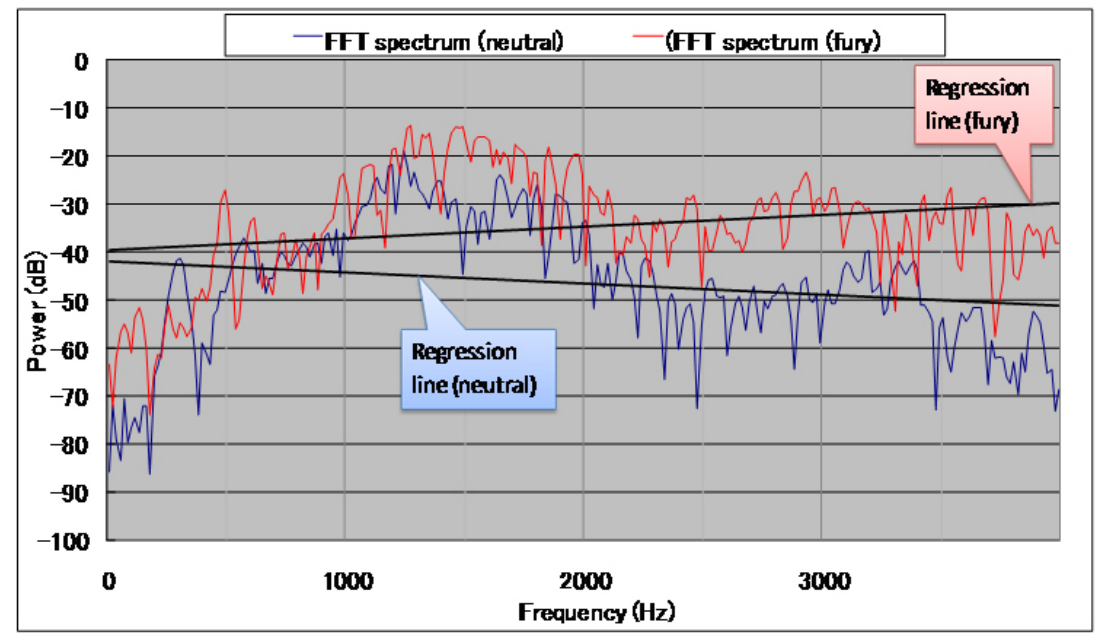

Figure 2: Spectra and their regression lines. Speaker: NN (female) 
emotion both uttered by female speaker NN.

Here, a spectral tilt parameter is defined as the primary coefficient of the regression-line function. As seen in this figure, the spectral tilt of "fury" speech is greater than that of "neutral".

\subsubsection{Summary of analysis results}

The results of analyses conducted so far showed that these spectral-tilt quantities were emotion-dependent, i.e., the spectral-tilt quantities for "anger" as well as "joy" increased significantly as the degree of each emotion became greater [20]. This confirms that the voice quality changes to the one whose higher-frequency band is more emphasized as the degrees of "anger" and "joy" increase.

Later, we conducted analysis of spectral-tilt quantities for "hot sadness" and "cold sadness" [16]. Figure 3 shows an example of analysis results. The results show that the spectral-tilt quantities for "hot sadness" tend to increase as the degree of each emotion becomes greater. In the same way as the cases of "anger" and "joy", this confirms that the voice quality changes to the one whose higherfrequency band is more emphasized as the degrees of "hot sadness" increases.

For "cold sadness", on the other hand, the tendency is opposite, i.e., the spectral-tilt quantities tend to decrease as the degree of each emotion becomes greater. This confirms that the voice quality changes to the one whose higher-frequency band is suppressed as the degrees of "cold sadness" increases.

These results for "sadness" are more or less speakerdependent since the ways of expressing "sadness" differ depending on speakers.

\section{SPECTRAL-TILT CONVERSION FILTER}

In this section, we define a transfer function of the spectral-tilt-conversion filters depicted in Figure 1.

The spectral-tilt quantity for "neutral" speech can be converted to that for emotional speech by using, for example, the following transfer function:

$$
S(z)=\left\{2-(1+\beta) z^{-1}\right\} /\left\{2-(1-\beta) z^{-1}\right\}
$$

where $\beta$ is a parameter that controls the amount of the spectral tilt. In the case that $\beta=1, S(z)=1-z^{-1}$, which coincides with the transfer function of primary differentiation. In this case, the spectral tilt increases by $6[\mathrm{~dB} /$ oct.] as frequency increases. In the case that $\beta=-1$, on the other hand, $S(z)=1 /\left(1-z^{-1}\right)$, which coincides with the transfer function of integration. In this case, the spectral tilt decreases by 6 [dB/oct.] as frequency increases. Furthermore, in the case that $\beta=0, S(z)=1$. In this way, if $\beta$ is varied in the range of $-1 \leq \beta \leq 1$, then the spectral tilt can be varied monotonically between -6 [dB/oct.] and 6 [dB/oct.].

\section{SPECTRAL-TILT VS. $\beta$ CHARACTERISTICS}

In this section, we describe measured results of spectraltiltquantitiesobtainedbyapplyingthespectral-tilt-conversion filter represented by Eq. (1) to natural "neutral" speech.

From our previous analysis results (Figure 4) [21], the predicted value of a spectral-tilt difference $\Delta \hat{S}_{\mathrm{tl}}$ can be formulated by Eq. (2) for any speakers in common. Here, a "spectral-tilt difference" means a spectral-tilt quantity of the emotional speech subtracted from that of the corre-

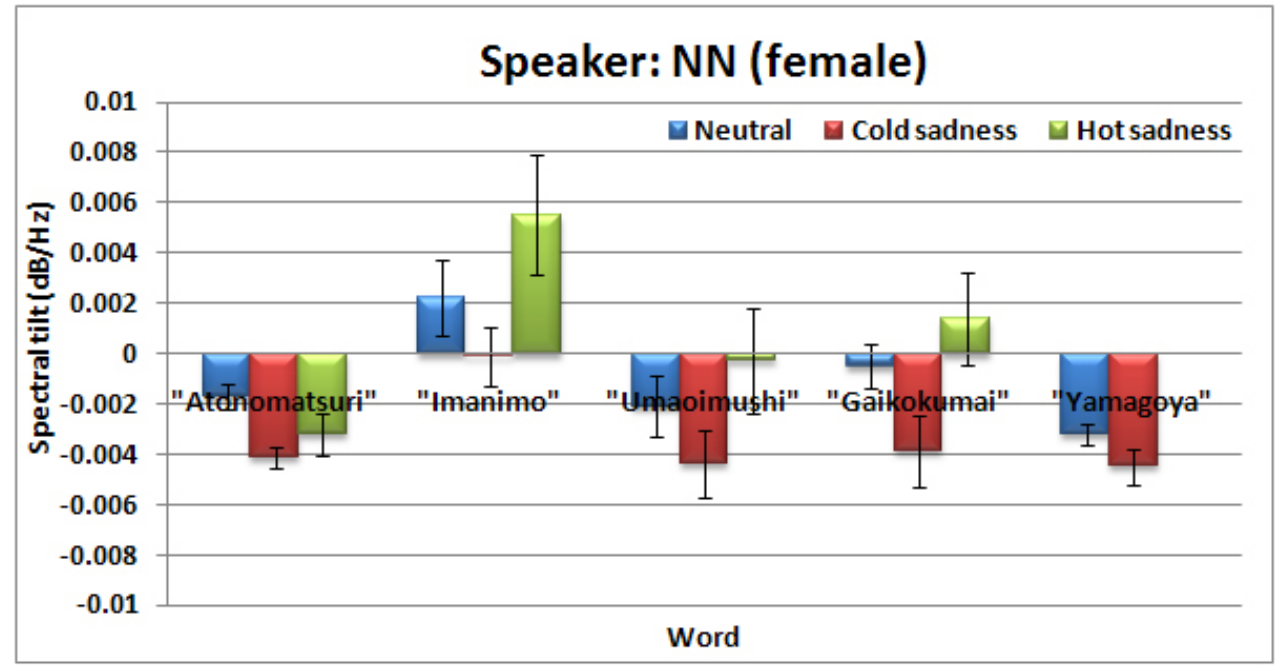

Figure 3: Results of FFT spectral-tilt analysis for "sadness" speech. Speaker: NN (female) [16] The height of each bar denotes a mean value of 5-sample spectral-tilt quantities and each error bar denotes a standard deviation. 
sponding neutral speech.

$$
\Delta \hat{S}_{\mathrm{tl}}=0.005 \beta
$$

Conversely, a spectral-tilt control parameter $\beta$ is obtained from the spectral-tilt difference $\Delta S_{\mathrm{tl}}$ by Eq. (3).

$$
\beta=200 \Delta S_{\mathrm{tl}}
$$

By knowing spectral-tilt values $S_{\mathrm{tl}}$ for various emotion types and degrees from the previous analysis results, we can obtain the corresponding $\beta$ values using Eq. (3). Figure 5 shows examples of spectral-tilt differences for various emotion types and degrees. While the quantities are speaker-dependent, general tendencies are mostly common to all speakers.

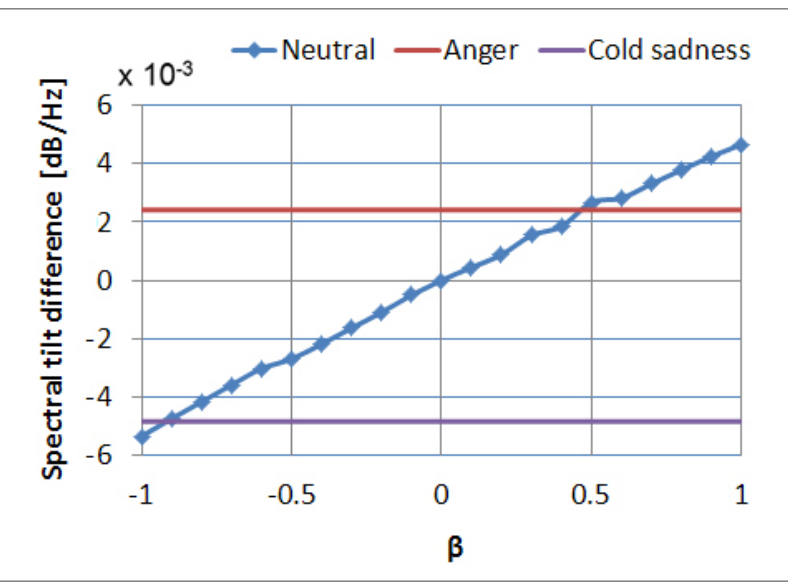

Figure 4: Spectral-tilt difference vs. $\beta$ [21]. Speaker: YF (male), /a/ in "ma" of word "yamagoya"

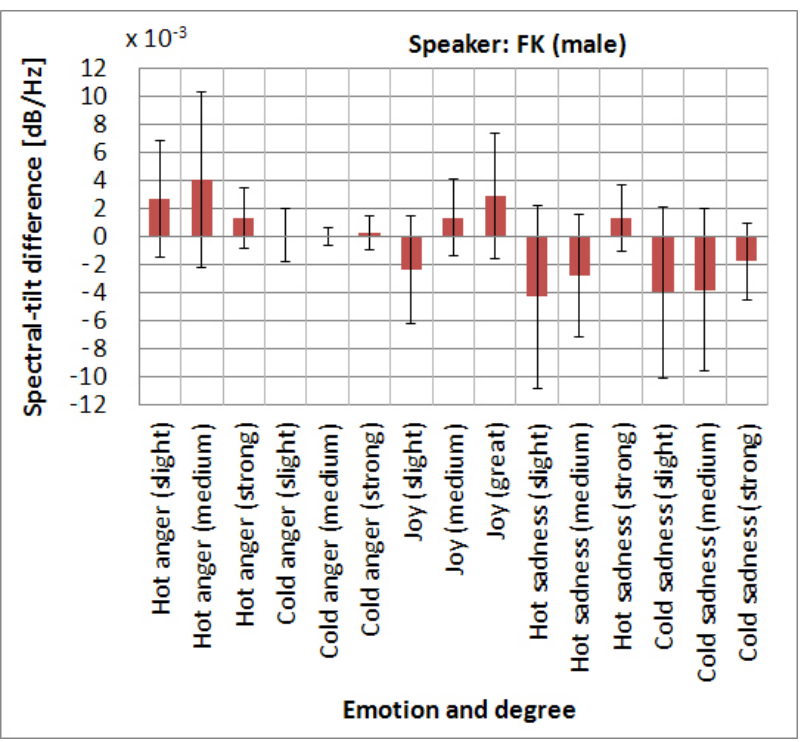

Figure 5: Spectral-tilt differences for various emotion types and degrees.

Speaker: FK (male), /a/ in the first "na" of word "manamana"

\section{APPLICATION TO SPEECH SYNTHESIS-BY- RULE}

As the first version of our rule-based emotional- speechsynthesis system [18], we adopted prosody-conversion as well as spectral-tilt-conversion rules. The system configuration is almost the same as that depicted in Figure 1, except that the excitation conversion is not introduced in the current version.

This time, we tried to synthesize variable degrees of "hot anger" and "cold sadness" speech.

\subsection{Prosody-Conversion Rule}

From observation of the data obtained from analyses, mora duration $d u$ (speech rate), minimum fundamental frequency $F_{0 \min }$, the magnitude of accent command $A_{\mathrm{a}}$, and speech power $P$ were converted according to the following rules. The rules were extremely simplified so that only qualitative features as listed Tables 1 and 2 were reflected in the rules because these prosodic-feature quantities were speaker-dependent and therefore accurate quantification was thought to be meaningless.

All the rules were determined emotion-by-emotion and the quantities were the functions of the degree of the emotion.

\subsubsection{Mora duration}

Mora duration was used to control speech rate.

(1) Hot anger

As a speech-rate model, the "increase-reduction" pattern, which was a pattern shown in speech uttered by male announcers as listed in Table 1, was adopted. The mora duration of "hot-anger" speech $d u_{\mathrm{ah}}$ is given by

$$
d u_{\mathrm{ah}}=d u_{\mathrm{n}}-c d_{\mathrm{ah}}(5-|\operatorname{deg}-5|)
$$

where $d u_{n}$ is the mora duration of the corresponding "neutral" speech and deg is the degree of the emotion. In the speech-synthesis program, deg is given as an integer number ranging from 0 to 9 . Number " 0 " denotes the weakest emotion (i.e., "neutral"), number "5" denotes midstrong emotion, and number "9" denotes the strongest emotion. $c d_{\text {ah }}$ is a constant that determines the strength of variation. $c d_{\mathrm{ah}}$ is experimentally determined as $c d_{\mathrm{ah}}=10$.

(2) Cold sadness

The mora duration of "cold-sadness" speech $d u_{\mathrm{sc}}$ is given by

$$
d u_{\mathrm{sc}}=d u_{\mathrm{n}}+c d_{\mathrm{sc}} d e g
$$

where $c d_{\text {sc }}$ is a constant that determines the strength of variation. $c d_{\mathrm{sc}}$ is experimentally determined as $c d_{\mathrm{sc}}=20$. 


\subsubsection{Minimum fundamental frequency}

As a prosodic feature parameter of fundamental frequencies, we used maximum fundamental frequency $F_{0 \max }$. On the other hand, we used minimum fundamental frequency $F_{0 \min }$ to generate $F_{0}$ contours by Fujisaki's model [4]. Since the relationship between $F_{0 \max }$ and $F_{0 \min }$ is rather complicated, it is difficult to resolve this mismatch. We temporarily used $F_{0 \text { min }}$ to control $F_{0}$ heights instead of $F_{0 \max }$.

(1) Hot anger

Minimum fundamental frequency of "hot anger" speech $F_{0 \text { minah }}$ is given by

$$
F_{0 \text { minah }}=F_{0 \operatorname{minn}}\left(1+c f_{\text {ah }} d e g\right)
$$

where $F_{0 \operatorname{minn}}$ is the minimum fundamental frequency of the corresponding "neutral" speech and $c f_{\text {ah }}$ is a constant that determines the strength of variation. $c f_{\text {ah }}$ is experimentally determined as $c f_{\text {ah }}=0.05$.

(2) Cold sadness

Minimum fundamental frequency of "cold sadness" speech $F_{0 \text { minsc }}$ is given by

$$
F_{0 \text { minsc }}=F_{0 \operatorname{minn}}\left(1-c f_{\mathrm{sc}} d e g\right)
$$

where $c f_{\text {sc }}$ is a constant that determines the strength of variation. $c f_{\mathrm{sc}}$ is experimentally determined as $c f_{\mathrm{sc}}=0.05$.

\subsubsection{Magnitude of accent command}

As another prosodic feature parameter of fundamental frequencies, we used the magnitude of accent command $A_{\mathrm{a}}$ that is necessary to generate $F_{0}$ contours by Fujisaki's model.

\section{(1) Hot anger}

The magnitude of accent command of "hot anger" speech $A_{\text {aah }}$ is given by

$$
A_{\text {aah }}=A_{\text {an }}\left(1+c a_{\text {ah }} d e g\right)
$$

where $A_{\text {an }}$ is the magnitude of accent command of the corresponding "neutral" speech and $c a_{\text {ah }}$ is a constant that determines the strength of variation. $c a_{\mathrm{ah}}$ is experimentally determined as $c a_{\mathrm{ah}}=0.05$.

\section{(2) Cold sadness}

The magnitude of accent command of "cold sadness" speech $A_{\text {asc }}$ is given by

$$
A_{\mathrm{asc}}=A_{\mathrm{an}}\left(1-c a_{\mathrm{sc}} d e g\right)
$$

where $c a_{\mathrm{sc}}$ is a constant that determines the strength of variation. $c a_{\mathrm{sc}}$ is experimentally determined as $c a_{\mathrm{sc}}=0.05$.

\subsubsection{Speech power}

(1) Hot anger

The decibel power of "hot anger" speech $d B P_{\text {ah }}$ is given by

$$
d B P_{\mathrm{ah}}=d b P_{\mathrm{n}}+c p_{\text {ah }} d e g
$$

where $d b P_{\mathrm{n}}$ is the decibel power of the corresponding "neutral" speech and $c p_{\text {ah }}$ is a constant that determines the strength of variation. $c p_{\text {ah }}$ is experimentally determined as $c p_{\text {ah }}=0.75$.

(2) Cold sadness

The decibel power of "cold sadness" speech $d B P_{\mathrm{sc}}$ is given by

$$
d B P_{\mathrm{sc}}=d b P_{\mathrm{n}}-c p_{\mathrm{sc}} d e g
$$

where $c p_{\mathrm{sc}}$ is a constant that determines the strength of variation. $c p_{\mathrm{sc}}$ is experimentally determined as $c p_{\mathrm{sc}}=0.75$.

\subsection{Spectral-tilt Conversion Rule}

The $\beta$ parameters were determined based on the quantities depicted in Figure 4, i.e., they were determined as simple functions of the degree of emotion as follows.

(1) Hot anger

$$
\beta=c t l_{\mathrm{ah}} \operatorname{deg}
$$

where $c t l_{\mathrm{ah}}$ is a constant that determines the strength of variation. $c t l_{\mathrm{ah}}$ is experimentally determined as $c t l_{\mathrm{ah}}=0.1$. (2) Cold sadness

$$
\beta=-c t l_{\mathrm{sc}} d e g
$$

where $c t l_{\mathrm{sc}}$ is a constant that determines the strength of variation. $c t l_{\mathrm{sc}}$ is experimentally determined as $c t l_{\mathrm{sc}}=0.1$.

\subsection{Examples of Synthesis Execution}

Figure 6 shows several examples of synthetic emotional speech waveforms and their $F_{0}$ contours in female voice. $F_{0}$ contours were generated by a special model based on Fujisaki's model, to which micro-prosody components were added [17].

It was observed from the figure that, as the degree of "hot anger" increased, the whole speech amplitudes (speech powers) were raised. The whole fundamental frequencies were also raised and the accent levels were emphasized. On the other hand, as the degree of "cold sadness" increased, the whole speech powers were lowered. The fundamental frequencies were also lowered and de-accented.

Changes in speech rate were also observed depending on the types and degrees of the emotions, i.e., as the degree of "hot anger" increased, the speech rate was first raised, and the degree further increased, it was reduced. On the other hand, as the degree of "cold sadness" increased, the speech rate was reduced. 


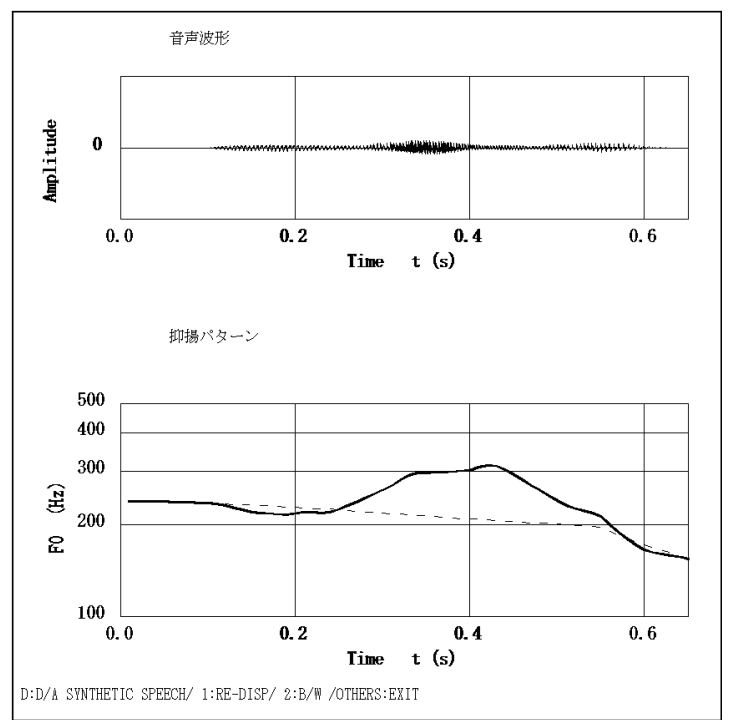

(a) Neutral

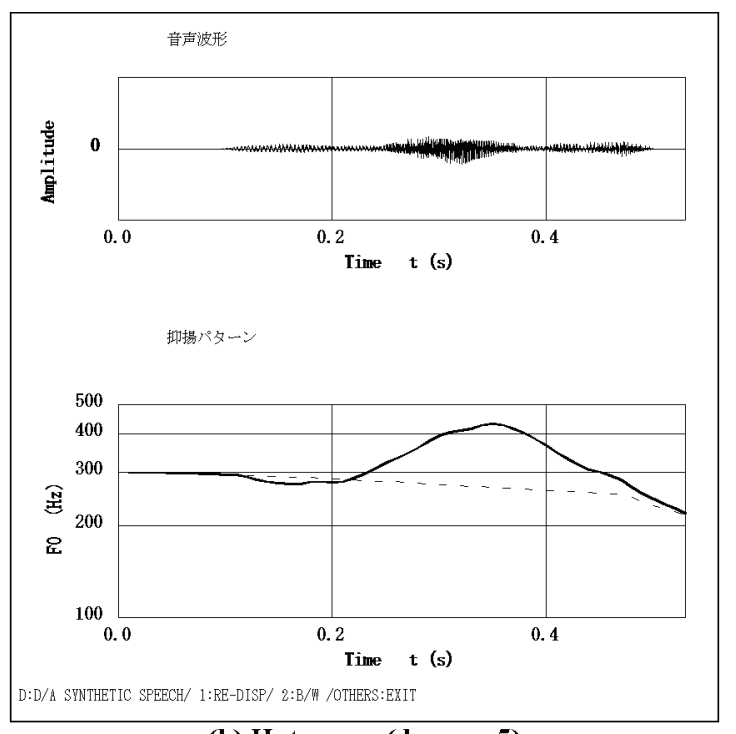

(b) Hot anger (degree=5)

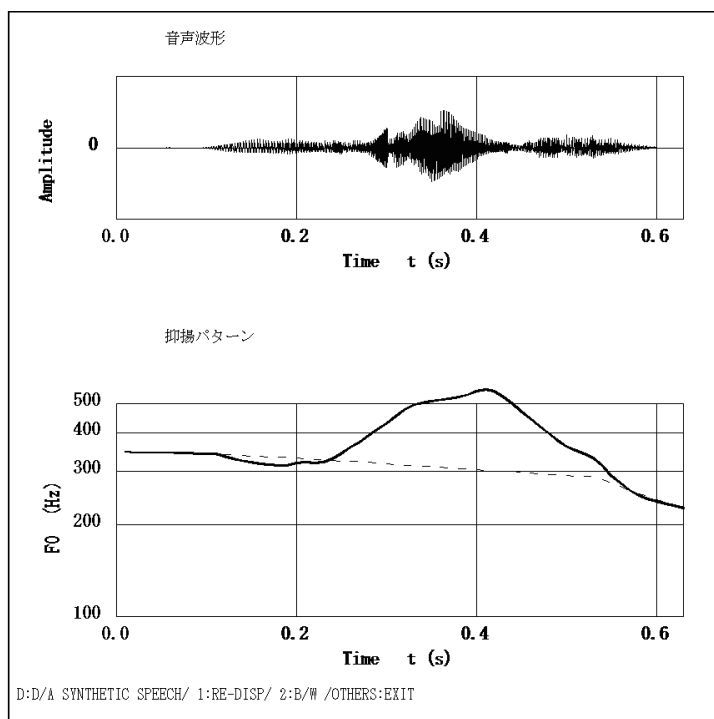

(c) Hot anger (degree=9)

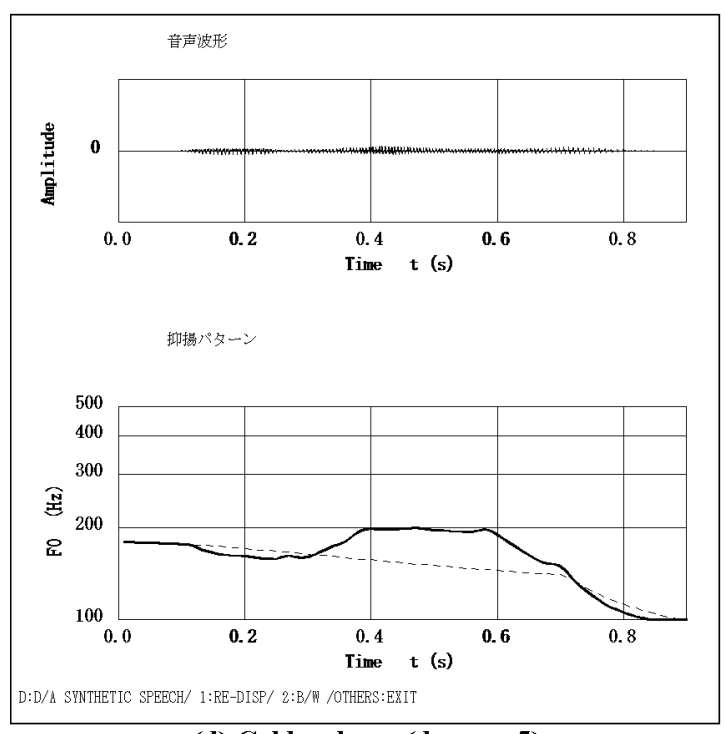

(d) Cold sadness (degree $=5$ )

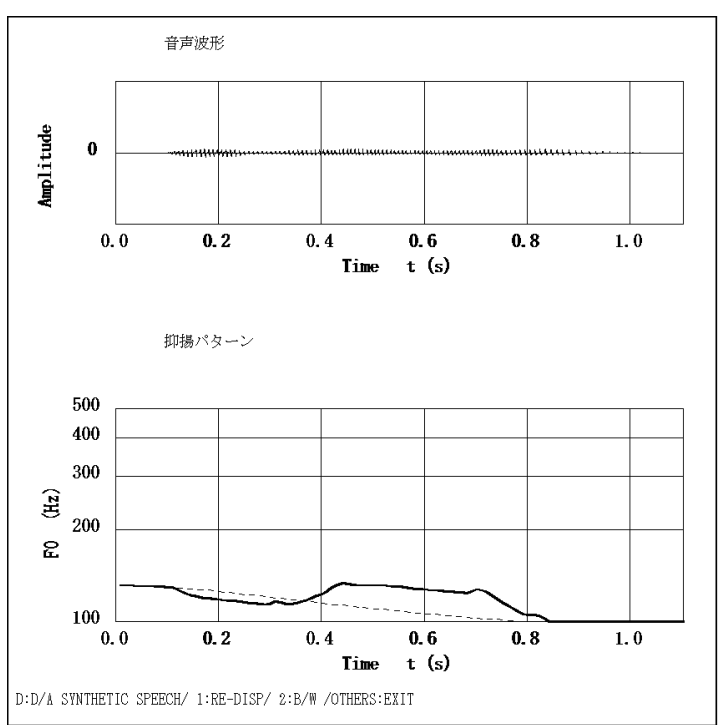

(e) Cold sadness (degree $=9$ )

Figure 6: Examples of synthetic speech waveforms (upper) and their $F_{0}$ contours (lower).

Word: "Iyayo" meaning "No"; voice: female; emotion: "neutral", "hot anger", and "cold sadness"; degree: 5 (middle) and 9 (strongest) 


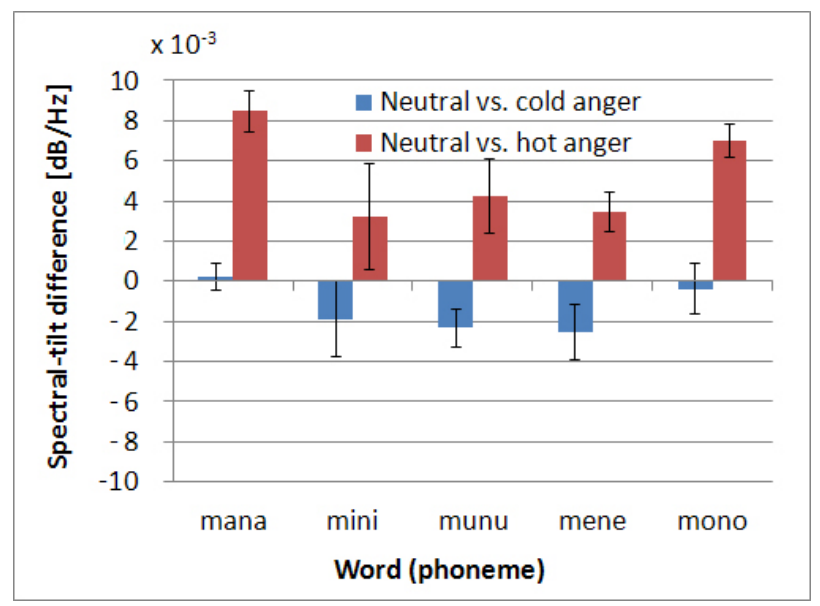

Figure 7: Phoneme-dependency of spectral- tilt differences. Speaker: AH (female)

Informal listening to these speech samples converted by the proposed method gave us impressions of those similar to natural emotional speech on prosody as well as voice quality, and the differences depending on the degrees of emotions were recognizable.

\section{DISCUSSION}

\subsection{Optimization of the Rules}

The set of rules proposed so far was only an example of realizing a means of emotional speech synthesis with dimensional representation. It is, however, not necessarily optimal. It is necessary to optimize rules. One way to explore optimal rules may be to determine the optimal parameters by a perception test.

\subsection{Phoneme-dependency of Spectral-tilt Quantities}

Since it is clear that a spectral tilt is affected by the formant structure of the speech, we need to take the effect of the phonemes into consideration.

Figure 7 shows the mean values (heights of the bars) and standard deviations (lengths of the error bars) of spectral-tilt differences for five words of "manamana", "minimini", "munumunu", "menemene", and "monomono" uttered by female speaker AH. The measuring points were the quasi-stable parts of the vowels in the first "na", "ni", "nu", "ne", and "no".

The common tendency was that the spectral-tilt quantities for "hot anger" were much greater than those for "neutral", whereas those for "cold anger" were nearly the same as or slightly smaller than those for neutral. Observing in details, however, the spectral-tilt quantities were phoneme-dependent. Introduction of phonemedependency into the voice-quality conversion rules will be a future work.

\subsection{Language-dependency of Spectral-tilt Features}

A question raised is whether or not the spectral-tilt conversion rule can be applied to other languages, e.g., English, Chinese, etc. Since the current version of the spectral-tilt conversion rule was derived only from Japanese speech samples, further analysis will be needed to know whether it can be applied to other languages. Predicted results are as follows. Since physiological features of expressing emotions are mostly common regardless of languages, the rule is expected to be applied to other languages. However, we knew that spectral-tilt quantities were phoneme-dependent as has been described above, so some modification will be needed when applying to other languages whose vowels are different from those of Japanese. Another point is that average voice quality may be language-specific, and therefore perception of voice quality may also be language-specific. E.g., "neutral" utterances spoken in a language may sound emotional to people whose native language is another. Confirmation of this question will also be left to future work.

\subsection{Application of a Spectral-tilt-conversion Filter to a Sentence}

This time, the spectral-tilt-conversion filter was applied to a single word. In the case of a sentence, emotional features may change every moment of the utterance. Further studies will be needed to know spectral-tilt features of a variety of sentences. The spectral-tiltconversion filter can be applied to a speech signal sample-by-sample. Therefore, once spectral-tilt features of a sentence are obtained, it is easy to form a filter adaptive to time-variation of spectral-tilt quantities.

\section{CONCLUSIONS}

This paper has proposed a Japanese speech synthesis system that is capable of expressing variable degrees of emotions based on prosody as well as voice-quality conversion. Among voice-quality features, we have placed a focus on a spectral tilt. Based on the emotiondependent features of spectral tilts known through our previous studies, we have formulated a transfer function that converts spectral-tilt quantities of "neutral" speech to those of emotional speech in various degrees.

Simple prosody and voice-quality-conversion rules have been proposed for synthesizing various degrees of "hot anger" and "cold sadness". We then have synthesized female-voice speech and confirmed that the qualitative prosodic features were reflected to the synthetic waveforms and $F_{0}$ contours according to the types and degrees 
of the emotions.

Informal listening to these speech samples converted by the proposed method has given us impressions of those similar to natural emotional speech on prosody as well as voice quality, and the differences depending on the degrees of emotions have been recognizable.

The results obtained through this study are promising in achieving synthesis of various types and degrees of emotional speech.

Future studies will be to determine the voice-quality conversion parameters by analyzing the features of several types and degrees of emotional speech, to synthesize such emotional speech using these conversion parameters, and to evaluate the emotional synthetic speech using the criterion of MOS (Mean Opinion Score) by a perception test.

Furthermore, the proposed work was derived from the results of analyses that were only applied to a single word, and therefore needs to be further examined in a sentence by Japanese.

\section{ACKNOWLEDGEMENTS}

The authors express their sincere appreciations to the actors and actresses at Gekidanseinenza Radio Theater for their help in uttering emotional speech.

This study was partly supported by the Project Research of the School of Biology Oriented Science and Technology, Kinki University No. 06-I-3, 2007-2009, and Grant-in-Aid for Scientific Research (C) "Interdisciplinary Research in Physiology and Acoustics on Active Characteristics of Kansei Evoked by Speech and Music Stimuli" from Japan Society for the Promotion of Science (No. 21500209), 2009-2012.

\section{REFERENCES}

1. Banse, R., and Scherer, K. R. (1996). Acoustic Profiles in Vocal Emotion Expression. Journal of Personality and Social Psychology, 70(3), pp.614-636.

2. Bänziger, T., and Scherer, K. (2007). Using Actor Portrayals to Systematically Study Multimodal Emotion Expression: The GEMEP Corpus. Affective Computing and Intelligent Interaction, pp. 476-487.

3. Campbell, N. (2007). Differences in the Speaking Styles of a Japanese Male According to Interlocutor; Showing the Effects of Affect in Conversational Speech. Computational Linguistics and Chinese Language Processing Vol.12, No.1, pp.1-16.

4. Fujisaki, H., Hirose, K. (1984). Analysis of voice fundamental frequency contours for declarative sentences of Japanese. J. Acoust. Soc. Jpn (E), 5(4), pp.233-242.

5. Hashizawa, Y., Takeda, S., Muhd Dzulkhiflee Hamzah., and Ohyama, G. (2004). On the Differences in Prosodic Features of Emotional Expressions in Japanese Speech according to the Degree of the Emotion. In Proc. 2nd Int. Conf. Speech Prosody, Nara, Japan, pp.655-658.

6. Hashizawa, Y., Takeda, S., Muhd Dzulkhiflee Hamzah., and Ohyama, G. (2005). Comparison of Prosodic Features of Emotional Speech Uttered by Announcers with Those Uttered by Radio Actors/Actresses According to the Degree of Emotion. In Proc. Spring Meet. Acoust. Soc. Jpn. 2-1-2, pp.207-208.

7. Hirose, K., Minematsu, N., and Kawanami, H. (2000). Analytical and perceptual study on the role of acoustic features in realizing emotional speech. In Proc. ICSLP2000, Beijing, China, pp.369-372.

8. Juslin, P. N., and Laukka, P. (2003). Communication of Emotions in Vocal Expression and Music Performance: Different Channels, Same Code? Psychological Bulletin, 129(5), pp.770-814.

9. Kasuya, H., Yoshizawa, M., and Maekawa, K. (2000). Roles of Voice Source Dynamics as a Converter of Paralinguistic Features. In Proc. ICSLP2000, Paper \#1283, Beijing, China.

10. Kitahara, Y., and Tohkura, Y. (1992). Prosodic control to express emotions for man-machine speech interaction. IEICE Trans. Fundamentals, E75-A(2), pp.155-163.

11. Kobayashi, Y., and Niimi, Y. (1993). On a prosodic information control method that reflects emotions in speech. In Proc. Fall Meet. Acoust. Soc. Jpn. 2-8-7, pp.233-234.

12. Liscombe, J., Venditti, J., and Hirschberg, J. (2003). Classifying Subjective Ratings of Emotional Speech Using Acoustic Features. In Proc. Eurospeech 2003, Geneva, pp.725-728.

13. In Proc. Interspeech 2010, Makuhari, Japan.

14. Scherer, K. R. (1986). Vocal Affect Expression: A Review and a Model for Future Research. Psychological Bulletin, 99(2), pp.143-165.

15. Schröder, M., and Grice, M. (2003). Expressing vocal effort in concatenative synthesis. In Proc. 15 th $\mathrm{ICPhS}$, Barcelona, pp.2589-2592.

16. Taguchi, Y., Uegaito, S., and Takeda, S. (2010). Analysis of Spectral-tilt Features of "Sadness" Speech-Research on Emotional-Speech Synthesis Based on Voice-Quality Conversion-. In Proc. 23rd Students' Meeting of High Information Technology 
Association (HIT), Amagasaki, Japan.

17. Takeda, S. (1990). A Model for Generating Fundamental Frequency Contours Considering Phoneme Fluctuation and Rules for Speech Synthesis. IEICE Trans. Fundamentals, J73-A, pp.379-386.

18. Takeda, S., Asakawa, Y., and Ichikawa, A. (1991). A Comparison of Synthetic Speech Quality Generated by the Residual- and Multipulse-Excited AnalysisSynthesis Methods. Electronics and Communications in Japan, Part 3, 74(4), pp.97-105.

19. Takeda, S., Yasuda, Y., Isobe, R., Kiryu, S., and Tsuru, M. (2008). Analysis of Voice-Quality Features of Speech that Expresses "Anger", "Joy", and "Sadness" Uttered by Radio Actors and Actresses. In Proc. Interspeech 2008, Brisbane, Australia, pp.2114-2117.

20. Takeda, S., Ueno, Y., Nakasako, N., Nakagawa, N., Tsuru, M., Isobe, R., and Kiryu S. (2010). SpectralTilt Features of Emotional Speech -Research on Emotional-Speech Synthesis Based on Voice-Quality Conversion-. In Proc. KEER2010, Paris, France, pp.1081-1090.

21. Takeda, S., Kabuta, Y., and Inoue, T. (2011). Calculation of spectral-tilt control parameters to realize speech synthesis with dimensional representation of emotions -Research on emotional-speech synthesis based on voice-quality conversion-. In Proc. Spring Meet. Acoust. Soc. Jpn. 2-p-21(a), pp.437-438.

22. Türk, O., and Schröder, M. (2008). A Comparison of Voice Conversion Methods for Transforming Voice Quality in Emotional Speech Synthesis. In Proc. Interspeech 2008, Brisbane, Australia, pp.2282-2285.

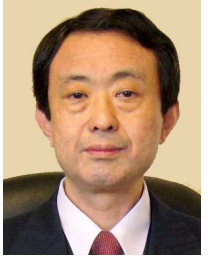

\section{Shoichi TAKEDA}

Shoichi Takeda received an M.E. in Mechanical Engineering for Production in 1974 and a Dr. Eng. degree in Electronic Engineering in 1991 from the University of Tokyo. In 1974, he joined Central Research Laboratory, Hitachi, Ltd., and attended Stanford University on a grant from the Rotary Foundation in 1974-1975. After returning to Japan he was engaged from 1975 to 1991 in research on recognition by artificial vision, speech synthesis-by-rule, etc. From 1991 to 2006, Dr. Takeda was with Teikyo Heisei University. Since 2006, Dr. Takeda has been professor at Kinki University and has been conducting research not only on speech synthesis-by-rule, but also on pathos (kansei) information processing including analysis of emotions by EEG or fNIRS measurements, and on digital archives for important cultural properties such as traditional Buddhist buildings. He is a senior member of the IEEE, and a member of the Japan Society of Kansei Engineering, the New York Academy of Sciences, the Institute of Electronics, Information and Communication Engineers, the Acoustical Society of Japan, etc. He received the Paper Award from the Japan Society of Kansei Engineering in 2006.

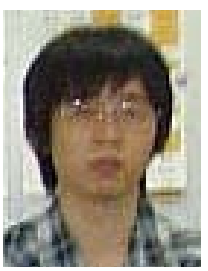

Yoshiki KABUTA

Yoshiki Kabuta received a B.E. in Electronic Systems and Information Engineering in 2011 from Kinki University. He is currently a graduate student in master's course at Kinki University and is engaging in research on speech synthesis-by-rule, specifically, on emotional speech synthesis.

Tomohiro INOUE

Tomohiro Inoue received a B.E. in Electronic Systems and Information Engineering in 2011 from Kinki University. From 2010 to 2011, he was engaged in research on speech synthesis-by-rule, specifically, on emotional speech synthesis.

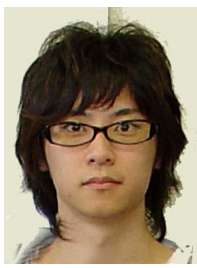

\section{Masashi HATOKO}

Masashi Hatoko received a B.E. in Electronic Systems and Information Engineering in 2012 from Kinki University. He is currently a graduate student in master's course at Kinki University and is engaging in research on speech synthesis-by-rule, specifically, on emotional speech synthesis and analysis of brain responses when uttering or listening to emotional speech. 